\title{
Der Name Gottes und das Problem interkultureller Übersetzbarkeit
}

\section{Die Namen der Völker}

Mozarts Freimaurerkantate KV 619, komponiert auf einen Text (und auf Bestellung) von E. H. Ziegenhagen im Sommer 1791, beginnt mit folgendem Rezitativ (Tenor):

Die Ihr des unermeßlichen Weltalls Schöpfer ehrt, Jehova nennt ihn, oder Gott -

Fu nennt ihn, oder Brahman Hört, hört Worte aus der Posaune des Allherrschers! Laut tönt von Erde, Monden, Sonnen ihr ewger Schall.

Der Text läßt dahingestellt, wie Gott eigentlich heißt und ob er überhaupt einen Namen hat. Darauf kommt es nicht an. Worauf es ankommt, ist, daß der Eine im Blick steht, der alles geschaffen hat und von dessen erhaltendem Schöpfertum das Universum kündet. Bei Mozart werden aber doch, im Unterschied zu Goethe, Namen genannt: Jehova, Gott, Fu, Brahman. Auch das ist ein Verfahren, das in antiken Texten oft begegnet. Der Lobpreis mit den Namen der Völker ist im Hellenismus und in der Spätantike ein beliebtes Verfahren hymnischer Preisung. Im p. Leiden I 384 wird in einer Weihezeremonie der Gott Iao-Sabaoth-Abrasax in einer ähnlichen Form angerufen:

Ich rufe dich nochmals an

wie die Ägypter: Phno eai Iabok, wie die Juden: Adonaie Sabaoth, wie die Griechen: König, der über alle herrscht, wie die ägyptischen hohen Priester: Verborgener, Unsichtbarer, der alle erblickt, wie die Parther: OYERTO [Großer auf der Erde], Herr über alles. ${ }^{1}$

1 Vgl. Abrasax. Ausgewählte Papyri religiösen und magischen Inhalts. Bd 1: Gebete. Hg. und erklärt von Reinhold Merkelbach u. a. Opladen: Westdeutscher Verlag 1990 (Abhandlungen der Rheinisch-Westfälischen Akademie der Wissenschaften. Sonderreihe Papyrologica Coloniensia, 17.1), S. 166f. Vgl. auch Erik Peterson: Eis theos. Epigraphische, formgeschichtliche und religionsgeschichtliche Untersuchungen. Göttingen: Vandenhoeck 
Eine magische Anrufung beginnt folgendermaßen:

Ich rufe dich an, der du das Universum umfaßt, in jeder Sprache und in jedem Dialekt. ${ }^{2}$

Es gibt so viele Namen Gottes, wie es verschiedene Sprachen gibt.

Der Lobpreis einer Gottheit mit den Namen und in den Sprachen der Völker ist ein Topos, der in zwei ganz verschiedenen Diskursen begegnet. Im magischen Diskurs, dem die beiden Beispiele entnommen sind, kommt es gerade auf die Namen an, weil ihre Lautgestalt eine beschwörende, 'präsentifizierende' Kraft besitzt; deshalb dürfen sie nicht übersetzt werden. Im philosophischen Diskurs dagegen besagt derselbe Topos das genaue Gegenteil: auf die Namen kommt es nicht an, weil alle Götter im letzten Grunde nur Namen eines Einzigen sind. Das ist der Glaube an ein Höchstes Wesen, das allen Göttern übergeordnet ist und zwar nicht nur als Schöpfer und Götterkönig, sondern auch als eine allen gemeinsame, in allen in verschiedener Brechung zur Erscheinung kommende Wesenheit. Dieses Wesen wird als Hypsistos, 'Höchster', angerufen ${ }^{3}$ oder auch mit der weitverbreiteten Heis Theos Formel ${ }^{4}$. Der Gedanke, daß der höchste Gott einer bestimmten Religion, eines Pantheons, alle anderen Götter dieses Pantheons in sich einbegreift, ist schon älter und läßt sich etwa im alten Ägypten bereits in der Ramessidenzeit, im 13. und 12. Jahrhundert v. Chr. belegen. Jetzt aber haben wir es mit der wahrhaft internationalen Idee eines Gottes zu tun, der die höchsten Wesen der verschiedenen Religionen in sich umfaßt. Die typische Form, in der diese Gottesidee zum Ausdruck kommt, sind Orakelsprüche, die die Identität verschiedener Höchster Götter in einem übergeordneten Einzigen Gott proklamieren:

\& Ruprecht 1926 (Forschungen zur Religion und Literatur des Alten und Neuen Testaments, NF 24), S. 254 für weitere Parallelen.

2 Papyri Graecae magicae. Die griechischen Zauberpapyri. Hg. und übersetzt von Karl Preisendanz. 2., verb. Aufl. mit Ergänzungen von Karl Preisendanz und Ernst Heitsch. Durchges. und hg. von Albert Henrichs. Stutgardiae: Teubner 1973, Bd II, S. 109, 119.

3 Zu Hypsistos vgl. Martin Nilsson, Harvard Theological Review, Vol. 56 (1963), S. 101120. Zu Hypsistos als Bezeichnung des Gottes der Juden und Übersetzung des hebr. El Elyon, vgl. Elias Joseph Bickerman: The Jews in the Greek Age. Cambridge/Mass.: Harvard University Press 1988, S. 263f.; Martin Hengel: Judentum und Hellenismus. Studien zu ihrer Begegnung unter besonderer Berücksichtigung Palästinas bis zur Mitte des 2. Jahrhunderts v. Chr. 3., durchges. Aufl. Tübingen: Mohr 1988 (Wissenschaftliche Untersuchungen zum Neuen Testament, 10), S. 545f; C. Colpe: Hypsistos. In: Der kleine Pauly. Lexikon der Antike auf der Grundlage von Pauly's Realencyclopädie der classischen Altertumswissenschaft bearb. und hg. von Konrat Ziegler u. a. Unveränd. Nachdr. d. Ausg. Stuttgart: Druckenmüller, 1964-1975. 5 Bde. München: Deutscher Taschenbuch Verlag 1979 (dtv, 5963), S. 1292-1293.

4 Peterson, Eis theos (wie Anm. 1); Otto Weinreich: Neue Urkunden zur Sarapis-Religion. Tübingen: Mohr 1919 (Sammlung gemeinverständlicher Vorträge und Schriften aus dem Gebiet der Theologie und Religionsgeschichte, 86). 
Der Eine ist Zeus, ist Hades, ist Helios, ist Dionysos.

Ein Gott in allen Göttern. ${ }^{5}$

Der Eine Zeus, der Eine Hades, der Eine Helios ist Sarapis. ${ }^{6}$

In diesem Orakelspruch wird Sarapis, der neue Gott, dessen Kult die Ptolemäer in der neugegründeten Stadt Alexandria eingerichtet hatten, in den Rang des einzigen allumfassenden Weltgottes erhoben. Auch Iao, der Gott der Juden, wird in einem dieser Orakelsprüche als Weltgott proklamiert: als Aion, die Welt als Zeit, hebräisch Olam, ist er Hades im Winter, Zeus im Frühling, Helios im Sommer und 'Abros Iao' im Herbst. ${ }^{7}$

Ganz besonders typisch ist dieses Motiv einer Anrufung mit den Namen der Völker für Isis. Das führt uns z. B. die wunderbare Szene vor Augen, mit der das 11. Buch der Metamorphosen des Apuleius von Madaurus, entstanden in der Zeit des Kaisers Mark Aurel, beginnt. Lucius, ein junger Mann, der durch neugieriges Pfuschen mit Magie in einen Esel verwandelt und in eine Fülle von Abenteuern verstrickt worden war, erwacht am Ufer des Mittelmeers vom Schein des über dem Meer aufgehenden Mondes. Die ersten 10 Bücher, in denen Apuleius sich eng an sein griechisches Original hält, erzählen seine burleske Leidensgeschichte. Mit dem 11. Buch aber verändert sich der Ton, und der Text verwandelt sich vom pikaresken Abenteuerroman zu einem tief religiösen Konfessions- und Mysterienroman, den A. D. Nock als "Höhepunkt jener Frömmigkeit" bezeichnete, "die aus den Mysterienreligionen hervorgegangen war"8. In der verwandelten Atmosphäre des 11. Buches, in die hinein der Held erwacht, gewinnt der Mondaufgang den Charakter einer Theophanie, so daß er das Gestirn mit folgenden Worten anredet (ich kürze ab): ${ }^{9}$

Himmelskönigin - ob du nun die allernährende Ceres bist, die Urmutter der Früchte [...], oder die himmlische Venus, die [...] im meerumfluteten Heiligtum von Paphos verehrt wird, oder die Schwester des Phoebus, die [...] jetzt im herrlichen Tempel von Ephesus angebetet wird, oder die dreigestaltige Proserpina, die $[\ldots]$ in mannigfachem Kult besänftigt wird [...] unter welchem Namen, nach wel-

5 Ps. Just. cohort. ad Graecos $15=$ Orph.fr. 239. Macrobius, Sat. I 18.17 zitiert den ersten Vers.

6 Handbuch der Altertumswissenschaft. Begr. von Iwan von Müller. Erw. von Walter Otto. Fortgef. von Hermann Bengtson. Abt. 5, Bd 2/2: Martin P. Nilsson: Geschichte der griechischen Religion. Tl. 2: Die hellenistische und römische Zeit. 4. Aufl., München: Beck 1988 , S. 573f.

7 Macrobius, Sat. I, 18, 20; vgl. Peterson, Eis theos (wie Anm. 1), S. 243f.; Hengel, Judentum und Hellenismus (wie Anm. 3), S. 476f. Vgl. auch die Inschrift Heîs Zeùs Sérapis Iaó (CIL II Suppl. 5665, Dunand 1975, 170).

8 Arthur Darby Nock: Conversion. The Old and the New in Religion from Alexander the Great to Augustine of Hippo. Oxford: Clarendon Press 1933 (Reprint 1963), S. 138.

9 Apuleius of Madauros: The Isis-Book (Metamorphoses, Book XI). Ed. with an Introduction, Translation and Commentary by John Gwyn Griffiths. Leiden: Brill 1975 (Etudes preliminaires aux religions orientales dans l'Empire Romain, 39), S. 70f., 114ff. 
chem Ritus, in welcher Gestalt man auch immer dich anrufen muß, hilf mir nun in meinem äußersten Elend [...]!

Lucius ruft eine göttliche, weibliche aber namenlose Macht, die er im Mond verkörpert glaubt, in Gestalt von vier möglichen Kandidatinnen solcher Verkörperung an, die ihm in den Sinn kommen: Ceres-Demeter, Venus-Aphrodite, Diana-Artemis und Proserpina-Persephone. Die spezifischen Namen, Epiklesen und Riten sind ihm viel weniger wichtig als die sich ihm im Mond offenbarende kosmische Macht. Die Göttin antwortet ihm im Traum und stellt sich ihm auf eine ganz ähnliche Weise vor. Auch sie beginnt ihre Selbstvorstellung mit einem Katalog von Namen:

Da bin ich, Lucius, durch dein Gebet gerüht, die Mutter der Natur [rerum naturae parens], die Herrin aller Elemente, erstgeborenes Kind der Zeit [saeculorum progenies initialis], die Höchste der Gottheiten, Königin der Toten, Erste der Himmlischen, die alle Götter und Göttinen in einer Erscheinung vereinigt [deorum dearumque facies uniformis], die ich mit meinem Wink über des Himmels lichte Gewölbe, des Meeres heilsame Lüfte und der Unterwelt vielbeweinte Stille gebiete, die alleinige Gottheit, welche unter mannigfacher Gestalt, verschiedenartigen Riten und vielerlei Namen der ganze Erdkreis verehrt, so nennen die Phrygier [...] mich Pessinuntia [...], die Athener [...] nennen mich Kekropische Athena, die Kyprier nennen mich paphische Venus, die Kreter Diktynna, die Sizilianer Ortygische Proserpina; die Eleusinier nennen mich Demeter, andere Hera, wieder andere Bellona und Hekate und Rhamnusia. Aber die Äthiopier und die Ägypter, die die ursprüngliche Lehre besitzen, ehren mich mit eigenen Bräuchen und nennen mich mit meinem wahren Namen Königin Isis.

Auch die Göttin verknüpft Namen und ethnische Gruppen. Die Namen sind wichtig, aber nur im Rahmen spezifischer Gruppen, die die Göttin in einer je bestimmten Form und mit je bestimmten Riten verehren. Aber neben diesen vielen ethnischen Namen hat sie auch einen 'wahren Namen', der aber nur bei den Völkern mit der ältesten und authentischsten Tradition in Gebrauch ist: bei den Ägyptern und den Äthiopiern. Die Erwähnung der Äthiopier in diesem Zusammenhang wird sich daraus erklären, daß Philae, der Hauptkultort der Isis im späten Ägypten, schon zu Nubien gerechnet wird.

Es gibt noch einige andere Isis-Texte aus Ägypten, die die Göttin mit den Namen der Völker preisen. Der früheste ist einer der vier Hymnen, die Isidorus von Narmuthis auf Pfeilern des Thermuthis-Tempels in Medinet Madi anbringen ließ (1. Jahrhundert v. Chr.): 10

Alle Sterblichen, die auf der grenzenlosen Erde leben, Thraker, Griechen und Barbaren, sprechen deinen schönen Namen aus, ein Name, hochgeehrt bei allen, [aber] jeder spricht in seiner eigenen Sprache, in seinem eigenen Land.

10 An dieser alten Kultstätte der ägyptischen Erntegöttin Renenutet oder (Th)ermuthis errichtete Ptolemäus Soter II einen Tempel für Isis-Thermuthis. 
Die Syrer nennen dich: Astarte, Artemis, Nanaia, die lykischen Stämme nennen dich Leto, die Herrin, die Thraker nennen dich Mutter der Götter und die Griechen "Hera vom Großen Thron", Aphrodite, Hestia die gütige, Rhea und Demeter.

Aber die Ägypter nennen dich Thiouis ${ }^{11}$ [weil sie wissen], daß du, die Eine, zugleich alle bist, die von den Völkern der Menschen angerufen werden. ${ }^{12}$

Ein dritter Text findet sich in einem Papyrus aus Oxyrhynchos. Er enthält einen langen Hymnus auf Isis, der mit einer sehr umfangreichen, aber leider sehr zerstörten Liste von Namen und Orten beginnt. ${ }^{13}$ Dort lesen wir etwa

[...] in Aphroditopolis die Eine [...], im Haus des Hephaistos [= Memphis] [-?-] chmuenis, die du in [-?-]ophis "Bubastis" genannt wirst, [-?-], in Letopolis Magna die Eine, [-?-] in Aphroditopolis im Prosopischen Gau Flottenführerin, vielgestaltige Aphrodite. Im Delta Gunstschenkerin, in Nithina im Gynaekopolitischen Gau Aphrodite; in Paphremis, Isis, Königin, Hestia, Herrin jeden Landes. [...] im saitischen Gau Siegreiche Athena [...]; in Sais, Hera, Königin, hochgewachsene; in Iseum, Isis; in Sebennytos Einsicht, Herrscherin, Hera, Heilige; in Hermupolis, Aphrodite, Königin, Heilige; [...] in Apis, Sophioa; in Leuke Akte, Aphrodite, Mouchis, Eseremphis; in Kynopolis im Busirites Praxidike; in Busiris, Gutes Geschick (Twxh agaqh); in Tanis, von anmutiger Gestalt, Hera [usw.]

Nach einer langen Liste solcher ägyptischer Ortsnamen und Isisnamen fährt der Text fort mit Namen außerägyptischer Orte und Länder wie Arabien, wo sie Große Göttin genannt wird, in Lykien "Leto", in Myra "Weise, Freiheit", in Knidos "die den Angreifer vertreibt, Entdeckerin", in Kyrene "Isis", in Kreta "Diktynnis", in Chalcedon "Themis", in Rom "Kriegerische", auf den Kykladen "Dreifache", auf Patmos "Junge [-?-]", auf Paphos "Begrüßte, Göttliche, Freundliche", auf Chios "Schreitende", in Salamis "Beobachterin", auf Zypern

11 Thiouis $=$ aeg. $t 3 w^{c} t$ kopt. TIOYI "die Eine", s. a. Vogliano: Primo Rapporto degli scavi condotti dalla Missione archeologica d' Egitto della R. universita di Milano nella zona di Madinet Madi. Milano 1936, S. 27-51, bes. S. 34.

12 Vera Frederika Vanderlip: The Four Greek Hymns of Isidorus and the Cult of Isis. Toronto: Hakkert 1972 (American Studies in Papyrology, 12), S. 18f.; Etienne Bernand: Inscriptions métriques de l'Égypte gréco-romaine. Paris: Belles Lettres 1969, Nr 175, S. 632ff.; Maria Totti: Ausgewählte Texte der Isis- und Sarapis-Religion. Hildesheim: Olms 1985 (Subsidia Epigraphica, 12), S. 76-82; Françoise Dunand: Le syncrétisme isiaque à la fin de l'époque hellénistique. In: Les syncrétismes dans les religions grecque et romaine. Colloque de Strasbourg, Bibliothèque des Centres d'Études supérieures spécialisés. Hg. von F. Dunand und Pierre Levêque. Paris: PUF 1973, S. 79-93. Zu Isidorus vgl. Drijvers, Vox Theologica, Jg 32 (1962), S. 139-50.

13 The Oxyrhynchus papyri. Ed. with Translations and Notes by Bernard P. Grenfell u. a. London: Egypt Exploration Fund 1915 (The Oxyrhynchus papyri, 11. Egypt Exploration Fund/Graeco-Roman Branch: Egypt Exploration Fund, Graeco-Roman Branch, 14), S. 196-202, Nr 1380. Vgl. van Groningen: De papyro Oxyrhynchita 1380. Groningen 1921; Nock, Conversion (wie Anm. 8), S. $150 \mathrm{ff}$. 
"Allfreigiebige" usw. usw., darunter auch fremde Namen: in Bamyke "Atargatis", bei den Indern "Maja", in Sidon "Astarte". Die Liste wird abgeschlossen mit einer auffallenden Formel: das schöne Leben aller Götter (qewn apántwn tò kalòn zwon).

Isis begreift alle Namen in sich. Daher wird sie auf einem Altar in Capua als "Eine die alles ist" bezeichnet: una qui es omnia. ${ }^{14}$ Isidorus ruft sie in einem seiner Hymnen an: du eine, die du alle bist (mounæ sy ei hapasai) ${ }^{15}$. Sie heißt geradezu "die Eine" $\left(t 3 w^{c} t-\right.$ THIOUIS). ${ }^{16}$

Seine reifste Ausprägung findet das Motiv des Lobpreises "in den Namen der Völker" in einem Epigramm des Ausonius, das im 18. Jahrhundert viel zitiert wurde und vermutlich das direkte Vorbild für Mozarts Textdichter abgegeben hat: ${ }^{17}$

Ogygiadae ${ }^{18}$ me Bacchum vocant,

Osiris Aegypti putant,

Mysi Phanacen nominant,

Dionyson Indi existimant,

Romana sacra Liberum,

Arabica gens Adoneum,

Lucaniacus Pantheum.

Die Söhne des Ogyges rufen mich Bacchus,

die Aegypter glauben mich Osiris,

die Mysier nennen mich Phanaces,

die Inder halten mich für Dionysos,

die römischen Riten nehmen mich für Liber,

14 Votiv-Inschrift aus Capua, 1. oder 2. Jh. n. Chr., CIL X $3800=$ Dessau ILS 4362; Sylloge inscriptionum religionis Isiacae et Sarapiacae. Collegit Ladislaus Vidman. Berolini: de Gruyter 1969 (Religionsgeschichtliche Versuche und Vorarbeiten, 28), Nr. 502: Te tibi una quae es omnia dea Isis. - Dunand, Le syncrétisme isiaque (wie Anm. 12), S. 82, Anm. 1.; Vincent Tran-tam-Tinh: Le culte des divinites orientales en campanie. En dehors de Pompei, de Stabies et d'Herculanum. Leiden: Brill 1972 (Etudes preliminaires aux religions orientales dans l'empire romain, 27), S. 41ff., 77, 199-234.

15 Hymnus des Isidorus aus Medinet Madi vgl. Vanderlip, The Four Greek Hymns (wie Anm. 12), S. 18f.; Bernand, Inscriptions métriques (wie Anm. 12), Nr 175, S. 632ff.; Totti, Ausgewählte Texte (wie Anm. 12), S. 76-82; Dunand, Le syncrétisme isiaque (wie Anm. 12), S. 79-93. Zu Isidorus vgl. Drijvers (wie Anm. 12).

16 Vanderlip, The Four Greek Hymns (wie Anm. 12), S. 31; vgl. hierzu C. H. Gordon: His name is 'One'. In: JNES, Vol. 29 (1970) S. 198f.

17 Epigramm XLVIII, überschrieben Mixobarbaron Liberi Patris Signo Marmoreo in Villa nostra omnium Deorum Argumenta habenti [Eine fremdländische Mixtur, auf eine Marmorstatue des Liber Pater in meinem Landhaus, die die Attribute mehrerer Götter aufweist]. Decimus Magnus Ausonius. With an English Translation by Hugh Gerard Evelyn White. 2 Vols, London, Cambridge/Mass.: Heinemann, Harvard University Press 1967/68 (The Loeb Classical Library), S. 186f. Epigramm Nr. XLIX ist eine griechische Fassung desselben Motivs.

18 White zufolge bezieht sich dieser Ausdruck auf die Thebaner, die Söhne des mythischen Stadtgründers Ogyges. 
das Volk der Araber für Adonis,

Lucaniacus $^{19}$ für den Allgott.

Eines gibt es in dieser kosmopolitischen Religion der Antike nicht, und das ist die Unterscheidung zwischen wahr und falsch. Alle Namen sind gleich treffend und gleich unzutreffend. Apuleius mit seinem Begriff des 'verum nomen' bildet hier eine Ausnahme; er steht schon halb auf dem Boden der Offenbarungsreligionen. Diese definieren sich über den Unterschied zwischen wahr und falsch und setzen alles andere zu sich in die Relation des Irrtums oder 'Heidentums'. Genau diese Unterscheidung wird in der Naturreligion der Aufklärung als unheilvoll empfunden. In den folgenden Abschnitten wollen wir einige Versuche betrachten, diese Unterscheidung rückgängig zu machen.

\section{Schall und Rauch}

Wer darf ihn nennen

Und wer bekennen:

Ich glaub Ihn!

Wer empfinden

Und sich unterwinden

Zu sagen: ich glaub ihn nicht!

Der Allumfasser,

Der Allerhalter,

Faßt und erhält er nicht

Dich, mich, sich selbst?

Wölbt sich der Himmel nicht dadroben?

Liegt die Erde nicht hierunten fest?

Und steigen freundlich blickend

Ewige Sterne nicht herauf?

Schau ich nicht Aug in Auge dir,

Und drängt nicht alles

nach Haupt und Herzen dir

Und weht in ewigem Geheimnis

Unsichtbar-sichtbar neben dir?

Erfüll davon dein Herz, so groß es ist,

Und wenn du ganz in dem Gefühle selig bist,

Nenn es dann, wie du willst:

Nenns Glück! Herz! Liebe! Gott!

Ich habe keinen Namen

Dafür! Gefühl ist alles;

Name ist Schall und Rauch,

Umnebelnd Himmelsglut. ${ }^{20}$

19 Das Landgut des Ausonius.

20 Goethe: Faust. Erster Teil. In: ders., Goethe's Werke. Vollständige Ausgabe letzter Hand. Stuttgart, Tübingen: Cotta 1828, Bd 12, S. 180f. 
Wenn wir von dem Begriff 'Gefühl' absehen, in dem sich Goethe als ein Autor des späten 18. Jahrhunderts zu erkennen gibt, dann bringt diese Antwort Fausts auf Gretchens berühmte Frage eine Überzeugung zum Ausdruck, die in den Kontext derselben hellenistischen und spätantiken Bildungsreligion gehört, auf den uns Mozarts Freimaurerkantate geführt hat. Servius meinte, daß es nur einen Gott gibt, dessen Namen je nach seinen Handlungsweisen und Zuständigkeiten wechselten. ${ }^{21}$ Varro (116-27 v. Chr.) hielt es für unnötig, zwischen Jupiter und Jahve zu unterscheiden, "weil es auf den Namen nicht ankäme, solange nur dieselbe Sache gemeint sei" (nihil interesse censens quo nomine nuncupetur, dum eadem res intelligatur) ${ }^{22}$. Porphyrius vertrat die Ansicht, daß die Namen der Götter auf bloßer Konvention beruhten. Celsus argumentierte in seinem Pamphlet gegen die Christen, Alethes Logos, daß "es keinen Unterschied macht, ob man Gott den 'Höchsten' nennt [Hypsistos] oder Zeus, oder Adonai, oder Sabaoth, oder Amun wie die Ägypter, oder Papaios, wie die Skythen"23. Auf den Namen kommt es nicht an, wenn der Gemeinte evident ist. Diese Evidenz war in einer Welt gegeben, in der sich alle Gebildeten einig waren, daß sie von einem einzigen Schöpfer erhalten, bewegt und beseelt werde. Die Auffassung von der Konventionalität und Übersetzbarkeit der göttlichen Namen beruhte auf natürlicher Evidenz, d. h. auf Erfahrungen, die jederzeit allen zugänglich waren. Solche Erfahrungen sind es, die Fausts rhetorische Fragen beschwören:

Der Allumfasser,

Der Allerhalter,

Faßt und erhält er nicht

Dich, mich, sich selbst?

Wölbt sich der Himmel nicht dadroben?

Liegt die Erde nicht hierunten fest?

In genau demselben Sinne verweist z. B. Seneca auf die sichtbare Einheit Gottes: "Dieses All, das du erblickst, und das Götter und Menschen umfaßt, ist Eines. Wir sind nur Glieder eines großen Körpers."24

Der klassischen griechischen Religion ist die Idee eines kosmischen Gottes fremd: sie kennt nur "Ressort-götter", aber keinen Weltgott (Nilsson ${ }^{25}$ ). Die Götter verschiedener Religionen lassen sich jedoch gerade über ihr Ressort gleichsetzen. Solche Götterübersetzung geht im zweisprachigen Mesopotamien bis in das 3. Jahrtausend zurück und wird im Laufe des 2. Jahrtausend auf

21 Eduard Norden: Agnostos theos. Untersuchungen zur Formengeschichte religiöser Rede. 7. Aufl., Neudr. der 1. Aufl. 1913, Stuttgart u. a.: Teubner 1996, S. 61.

22 Augustin: De consensu evangelist, 1, 22, 30 and 23, 31 PL 34, 1005f = Varro fr. I, 58b, siehe Hengel, Judentum (wie Anm. 3), S. 472.

23 Ap. Origenes c. Cels. I 24, V 41 (45); siehe Hengel, Judentum (wie Anm. 3), S. 476.

24 Uvo Hölscher: Empedokles und Hölderlin. Frankfurt a. M.: Insel-Verlag 1965, S. 48.

25 Nilsson, Geschichte der griechischen Religion (wie Anm. 6), S. 295. 
viele Sprachen und Völker des vorderen Orients ausgedehnt. Der Sonnengott der einen Religion ist derselbe wie der Sonnengott einer anderen Religion. Plutarch bringt in seinem Traktat über Isis und Osiris diese allgemeine Überzeugung auf den Begriff. Hinter den verschiedenen Götternamen, schreibt er, stehen immer dieselben kosmischen Phänomene: die Sonne, der Mond, der Himmel, die Erde, das Meer usw. Da alle Menschen in ein und derselben Welt leben, verehren sie dieselben Götter, die die Herren dieser Welt sind. ${ }^{26}$ In der späteren Antike verdichtet sich das Bewußtsein von der interkulturellen Identität der kosmischen Götter zur übergreifenden Einheit des Einen Gottes, der Schöpfer, Erhalter, Beleber und Inbegriff der Welt ist.

Auf diese Religion des einen, alles umgreifenden Gottes 'mundus' hat Malesherbes den Begriff 'Kosmotheismus' geprägt. In seiner Ausgabe der historia naturalis von Plinius dem Älteren kommentierte er eine für diese Religion besonders typische Stelle - mundum, et hoc quodcumque nomine alio coelum appellare libuit, cujus circumflexu teguntur cuncta, numen esse credi par est mit dem Vorschlag, Plinius nicht einen Atheisten zu nennen, sondern "un Cosmo-théiste, c'est à dire quelqu'un qui croit que l'univers est Dieu"27. Malesherbes hätte keinen glücklicheren Begriff finden können, um das gemeinsame Element der altägyptischen, neoplatonischen, stoischen, hermetischen ... Religion hervorzuheben, das im Spinozismus des späten 18. Jahrhunderts, vermittelt durch alchemistische und kabbalistische Unterströme, zurückkehrt. ${ }^{28}$

26 Plutarch: De Is. c. 67 , p. $377 \mathrm{E}$ ff.: "[...] nor do we regard the gods as different among different peoples nor as barbarian and Greek and as southern and northern, but just as the sun, moon, heaven, earth and sea are common to all, though they are given various names by the varying peoples, so it is with the one reason [logos] which orders these things and the one providence which has charge of them, and the assistant powers which are assigned to everything: they are given different honours and modes of address among different peoples according to custom, and they use hallowed symbols [...]." (Plutarch's De Iside et Osiride. Ed. with an Introduction, Translation and Commentary by J. Gwyn Griffiths. Cardiff: University of Wales Press 1970, S. 223f.)

27 Emmanuel J. Bauer: Das Denken Spinozas und seine Interpretation durch Jacobi. Bern. u. a.: Lang 1989 (Europäische Hochschulschriften, 20/270), S. 234ff.

28 Vgl. Friedrich Heinrich Jacobi: Über die Lehre des Spinoza in Briefen an Herrn Moses Mendelssohn. In: ders., Werke. Hg. von Friedrich Roth u. a. Darmstadt: Wissenschaftliche Buchgesellschaft 1968, Bd 4/1, S. 1-253 hier 217-219; Hermann Timm: Gott und die Freiheit : Studien zur Religionsphilosophie der Goethezeit. Frankfurt a. M.: Klostermann 1974 (Studien zur Philosophie und Literatur des neunzehnten Jahrhunderts, 22), S. 226ff. Schon 1699 benutzte Johann Georg Wachter den Ausdruck "vergötterte Welt" mit Bezug auf Spinoza. Vgl. Gershom Scholem: Die Wachtersche Kontroverse. In: Spinoza in der Frühzeit seiner religiösen Wirkung. $\mathrm{Hg}$. von Karlfried Gründer und Wilhelm SchmidtBiggemann. Heidelberg: Lambert Schneider 1984 (Wolfenbütteler Studien zur Aufklärung, 12), S. $15-25$ bes. S. 15 . 


\section{III. 'Der ich bin' und 'Was da ist' - Jehovah sive Isis}

Karl Leonhard Reinhold, dem wir uns als nächstes zuwenden wollen, stand Mozart nicht ganz fern. ${ }^{29} \mathrm{Er}$ gehörte derselben Wiener Freimaurerloge an, in der auch Mozart ein und ausging, der Loge 'Zur wahren Eintracht' ${ }^{\prime 30}$. Allerdings verlie $ß$ er Wien, bevor Mozart dem Orden beitrat. 1786 publizierte er zwei lange Artikel über "Die hebräischen Mysterien" im Wiener Journal für Freymaurer, die er 1788, inzwischen als außerordentlicher Professor für Philosophie nach Jena berufen, als Buch herausbrachte. ${ }^{31}$ In diesem Buch vertritt er die These, daß der hebräische Name Gottes, JHWH, und der Name der Isis dasselbe bedeuten. Dabei stützt er sich nicht auf die Namen selbst, sondern auf deren Ausdeutungen in den heiligen Texten. Für das Tetragramm zieht er Exodus 3,14 heran - "Ich bin/werde sein, der ich bin/sein werde" - und für Isis die berühmte, von Plutarch und Proklus überlieferte Inschrift auf dem verschleierten Bildnis zu Sais: "Ich bin alles, was da ist".

"Wem aus uns, meine Brüder!" ruft er aus,

sind endlich die alten ägyptischen Inschriften unbekannt; die eine auf der Pyramide zu Sais: Ich bin alles, was ist, war und seyn wird, meinen Schleier hat kein Sterblicher aufgehoben; und jene andere unter der Bildsäule der Isis: Ich bin, was da ist? Wer aus uns, meine Brüder! versteht nicht den Sinn dieser Worte so gut, als ihn vormals der ägyptische Eingeweihte verstehen mußte, und weiß nicht, daß damit das wesentliche Daseyn, die Bedeutung des Wortes Jehovah, beynahe wörtlich ausgedrückt ist? ${ }^{32}$

Plutarch und Proklus kennen allerdings nur eine Inschrift. Die zweite, eine Kurzfassung der ersten, scheint auf einem Mißverständnis zu beruhen. Sie begegnet bereits bei Voltaire, den Reinhold hier paraphrasiert. ${ }^{33}$ Sie macht die

29 Zu Reinhold vgl. Gerhard W. Fuchs: Karl Leonhard Reinhold - Illuminat und Philosoph. Eine Studie über den Zusammenhang seines Engagements als Freimaurer und Illuminat mit seinem Leben und philosophischen Wirken. Frankfurt a. M. u. a.: Lang 1994 (Schriftenreihe der Internationalen Forschungsstelle "Demokratische Bewegungen in Mitteleuropa 1770-1850", 16).

30 Mozart war seit 1785 Mitglied einer Tochterloge, Zur Wohltätigkeit. Er war jedoch mit den Mitgliedern der Hauptloge Zur Wahren Eintracht, zu denen Josef Haydn, Baron van Swieten, Joseph von Sonnenfels und andere Freunde Mozarts gehörten, eng verbunden. Für den Meister vom Stuhl, Ignaz v. Born (der auch Modell für den Sarastro der Zauberflöte gewesen sein soll) komponierte Mozart die Kantate "Die Maurerfreude" KV 471. Ab 1786 wurden die Wiener Logen reduziert und in der Loge Zur Gekrönten Hoffnung zusammengefaßt.

31 Br[uder] Decius [d. i. Karl Leonhard Reinhold]: Die Hebräischen Mysterien oder die älteste religiöse Freymaurerey. In zwey Vorlesungen gehalten in der ... zu ****. Leipzig: Göschen 1788. - Decius war Reinholds Geheimname als Mitglied des Illuminatenordens (vgl. Fuchs, Karl Leonhard Reinhold [wie Anm. 29], S. 25). Das Buch über die Hebräischen Mysterien wird bei Fuchs nicht erwähnt.

32 Reinhold, Die Hebräischen Mysterien (wie Anm. 31), S. 54.

33 De rites égyptiens, in: Essay sur le moeurs des peuples, § XXII (๕Euvres de Voltaire. Hg. von M. Beuchot. Paris 1829, Bd XV, S. 102-106. 
Parallele noch schlagender, um die es hier geht: "Ich bin der ich bin" - "Ich bin was da ist". Schon Vico hatte das ascher ahjoeh von Exodus 3,14 als "der ich bin und was da ist" wiedergegeben. ${ }^{34}$ Reinhold folgt hier einer alten Tradition, die letztlich auf die Septuaginta zurückgeht. Dort wird die Selbstvorstellung Gottes wiedergegeben als "Ego eimi ho on", "Ich bin der Seiende", worunter Reinhold im Sinne der scholastischen Tradition "das wesentliche Daseyn" versteht.

Schon in einem der als "sibyllinische Orakel" gesammelten Orakelsprüche wird Exodus 3,14 im Sinne eines universalen Allgotts ausgelegt, der die Welt nicht nur geschaffen hat, sondern sich geradezu in ihr verkörpert:

Ich bin der Seiende, erkenne dies in deinem Geist: ich legte den Himmel an als Gewand, ich bekleidete mich mit dem Ozean, die Erde ist der Grund meiner Füße, die Luft umgibt mich als Körper und die Sterne umkreisen mich. ${ }^{35}$

Mit diesem Text sind wir wiederum auf den hellenistischen und spätantiken Kosmotheismus verwiesen, den wir in den ersten beiden Abschnitten betrachtet haben.

Der Kosmotheismus ist allerdings die genaue Umkehrung dessen, worum es im hebräischen Monotheismus ursprünglich und eigentlich geht. "Die Luft ist mein Körper, Himmel und Ozean sind mein Gewand": so spricht der kosmische Gott, "le dieu cosmique", wie André-Jean Festugière ihn treffend genannt hat ${ }^{36}$, aber nicht der Gott der Bibel. Es ist für uns heutige schwer begreiflich, wie der Unterschied zwischen den Aussagen "Ich bin, der ich bin" und "Ich bin das All" derart verwischt werden konnte, sowohl im Altertum in dem sibyllinischen Orakeltext, als auch im 18. Jahrhundert. Die eine Aussage ist vielmehr die Negation der anderen. "Ich bin der ich bin" ist die Verweigerung jeder kosmischen Identifikation. So spricht ein Gott, der auf nichts außerhalb seiner verweist, sondern allen kosmischen Identifikationen den Boden entzieht. Dieser Satz ist die Negation des Kosmotheismus. ${ }^{37}$ Mit der kosmischen Referenz wird aber zugleich auch der interkulturellen Übersetzbarkeit der Boden entzo-

34 [Giambattista] Vico: Selected Writings. Ed. and translated Leon Pompa. Cambridge: Cambridge University Press 1982, S. 53 (On the ancient wisdom of the Italians, Kap. II).

35 Abrasax (wie Anm. 1), Bd 2 [1991]: Gebete, S. 131.

36 Andre-Jean Festugière: La révélation d'Hermès Trismégiste. Avec un appendice sur l'hermetisme arabe par Louis Massignon. Vol. II: Le dieu cosmique. Paris: Lecoffre 1949 (Etudes Bibliques).

37 Zu Ex 3,14 vgl. u. a. Oskar Grether: Name und Wort Gottes im Alten Testament. Gießen: Töpelmann 1934 (Zeitschrift für die alttestamentliche Wissenschaft. Beiheft 64), S. $3 \mathrm{ff}$.; Wolfram von Soden: Bibel und Alter Orient. Altorientalische Beiträge zum Alten Testament. Berlin: de Gruyter 1985, S. 78-88; Georg Fohrer: Geschichte der israelitischen Religion. Berlin: de Gruyter 1969, S. 63ff.; Johannes C. de Moor: The Rise of Yahwism. The Roots of Israelite Monotheism. Leuven: University Press 1990 (Bibliotheca Ephemeridum theologicarum Lovaniensium, 91), S. 175, 237ff. 
gen. Jahwe ist bei aller Transzendenz zugleich ein dezidiert nationaler Gott. Er ist das genaue Gegenteil des kosmischen Gottes, den die römische und griechische Spätantike als den Einen über allen nationalen Göttern verehrte.

Wieder werden wir, von einem Gedanken des späten 18. Jahrhunderts ausgehend, auf die Antike geführt. Die kosmotheistische Auslegung des biblischen Gottesnamens, und damit die Grundlage von Spinozas deus sive natura (sive Isis) geht auf dieselbe hellenistisch-spätantike Religiosität zurück, wie wir sie im ersten Abschnitt betrachtet haben.

$\mathrm{Zu}$ Reinholds ersten Lesern gehörte sein Freund und Kollege an der Jenaer Fakultät, Friedrich Schiller, der sich von Reinholds Buch zu seiner Ballade "Das verschleierte Bildnis zu Sais" sowie zu seinem Essay "Die Sendung Moses" inspirieren ließ. Schillers Essay ist eine Zusammenfassung von Reinholds Buch. Insbesondere fasziniert ihn der Aspekt des Geheimnisses und der Einweihung. Ägypten ist das Land eines esoterischen Monotheismus.

Da Ägypten der erste kultivierte Staat war, den die Geschichte kennt, und die ältesten Mysterien sich ursprünglich aus Ägypten herschreiben, so war es aller Wahrscheinlichkeit nach hier, wo die erste Idee von der Einheit des höchsten Wesens zuerst in einem menschlichen Gehirne vorgestellt wurde. ${ }^{38}$

Diese Einsicht blieb in Ägypten aber Sache weniger Eingeweihter. Zu tief hatte sich bereits die Vielgötterei festgesetzt, so daß mit der Abschaffung des alten Aberglaubens das ganze Staatsgebäude in sich zusammengebrochen wäre.

Man fand also für besser, die neue gefährliche Wahrheit zum ausschließenden Eigenthum einer kleinen geschlossenen Gesellschaft zu machen, diejenigen, welche das gehörige $\mathrm{Ma} \beta$ von Fassungskraft dafür zeigten, aus der Menge hervorzuziehen und in den Bund aufzunehmen, und die Wahrheit selbst, die man den unreinen Augen entziehen wollte, mit einem geheimnisvollen Gewand zu umkleiden, das nur derjenige wegziehen könnte, den man selbst dazu fähig gemacht hätte. ${ }^{39}$

Dieses Gewand ist die Hieroglyphenschrift, die als eine Geheimschrift verstanden wird. Sie schützt die gefährliche monotheistische Wahrheit, die eine Art politischen Sprengstoff darstellt, und erregt zusammen mit komplizierten Zeremonien eine "sinnliche Feierlichkeit"40, die die Seele des Initianden durch emotionale Erregung zur Aufnahme der Wahrheit bereit machen soll. Nur ganz Wenigen, und nur am Ende eines oft jahrzehntelangen Einweihungsprozesses wird die Wahrheit enthüllt.

38 Vgl. Lukian: De Dea Syria, Kap. 2: "Die Ägypter sollen die ersten von allen uns bekannten Völkern gewesen sein, die sich Begriffe von den Göttern gemacht haben. Nicht viel später hörten die Syrer von den Ägyptern die Rede über die Götter und errichteten Tempel und Heiligtümer". Schiller: Die Sendung Moses. In: ders., Sämtliche Werke, Bd IV: Historische Schriften. Hg. von H. Koopmann. München 1968, S. 737-757, hier S. 743.

39 Ebd., S. 744.

40 Ebd. 
Unter einer alten Bildsäule der Isis las man die Worte: "Ich bin, was da ist" und auf einer Pyramide zu Sais fand man die uralte merkwürdige Inschrift: "Ich bin alles was ist, war und was sein wird, kein sterblicher Mensch hat meinen Schleier aufgehoben". [...] In dem Hymnus, den der Hierophant [...] dem Einzuweihenden vorsang, war dies der erste Aufschluß, der über die Natur der Gottheit gegeben wurde: Er ist einzig von ihm selbst, und diesem Einzigen sind alle Dinge ihr Dasein schuldig. ${ }^{41}$

Beethoven, dem Matthias Artaria die Lektüre von Schillers Aufsatz empfohlen hatte, war von diesen Sätzen so beeindruckt, daß er sie sich nicht nur abschrieb, sondern unter Glas rahmen ließ und sich auf seinen Schreibtisch stellte. ${ }^{42}$ In solchen Sätzen, die man für den Ausdruck urältester ägyptischer Weisheit hielt, erkannte die Zeit ihr eigenstes Anliegen wieder: eine Religion der Vernunft und der Natur.

Im selben Jahr wie Schillers Essay erschien Kants Kritik der Urteilskraft. Er geht darin zwar nur in einer Fußnote auf die Inschrift von Sais ein, aber seine Deutung der Inschrift als Inbegriff des 'Erhabenen' ist berühmt geworden:

vielleicht ist nie etwas Erhabeneres gesagt oder ein Gedanke erhabener ausgedrückt worden als in jener Aufschrift über dem Tempel der Isis [der Mutter Natur]: "Ich bin alles was da ist, was da war und was da sein wird, und meinen Schleier hat kein Sterblicher aufgedeckt." Segner benutzte diese Idee, durch eine sinnreiche, seiner Naturlehre vorgesetzte Vignette, um seinen Lehrling, den er in diesen Tempel einzuführen bereit war, vorher mit dem heiligen Schauer zu erfüllen, der das Gemüth zu feierlicher Aufmerksamkeit stimmen soll. ${ }^{43}$

Kant verwendet Schillers Sprache der Initiation - "heiliger Schauer", "feierliche Aufmerksamkeit" -, um die Verbindung herzustellen zwischen der Erfahrung des Erhabenen und der Einweihung in eine ungewöhnliche, ja überwältigende Eröffnung. Segners Vignette und der Ikonographie des verschleierten Bildes zu Sais ist Pierre Hadot in einer meisterhaften Studie nachgegangen ${ }^{44}$. Er konnte zeigen, daß diese in vielen Titelkupfern naturkundlicher Werke benutzte Szene als Allegorie der Naturwissenschaft ver-

41 Schiller, Sendung Moses (wie Anm. 38), S. $745 f$.

42 Vgl. Anton Schindler: Biographie von Ludwig van Beethoven. 3., neubearb. und verm. Aufl., 2 Teile, Münster: Aschendorff 1860, S. 161. Beethovens in Faksimile wiedergegebener Text lautet: "Ich bin, was da ist / Ich bin alles, was ist, was war, und was seyn wird, kein sterblicher Mensch hat meinen Schleyer aufgehoben / Er ist einzig von ihm selbst, u. diesem Einzigen sind alle Dinge ihr Daseyn schuldig." Vgl. auch Erhart Graefe: Beethoven und die ägyptische Weisheit. In: Göttinger Miszellen, Jg 2 (1971), S. 19-21.

43 Immanuel Kant: Kritik der Urteilskraft. In: ders., Werke in 10 Bänden. Hg. von W. Weischedel. Darmstadt 1968, Bd 8, S. 417.

44 Pierre Hadot: Zur Idee der Naturgeheimnisse. Beim Betrachten des Widmungsblattes in den Humboldtschen "Ideen zu einer Geographie der Pflanzen". Wiesbaden: F. Steiner 1982 (Abhandlungen der Geistes- und Sozialwissenschaftlichen Klasse / Akademie der Wissenschaften und der Literatur, Jg 1982, Nr 8). 
standen wurde, entweder im optimistischen Sinne der endlich gelingenden Entschleierung oder im Sinne eines epistemologischen Pessimismus, der die volle Wahrheit für letztlich unerreichbar hält. Den heiligen Schauer des Erhabenen gibt jedoch nur Heinrich Füßlis außerordentliches Titelkupfer zu Erasmus Darwins Gedicht "The Temple of Nature" (1808) wieder. ${ }^{45}$ Hier wird eine wirkliche Einweihungsszene gezeigt. Eine Hierophantin enthüllt mit abgewendetem Gesicht eine Statue der Isis im Typus der Ephesischen Diana multimammia, die von einer knienden Initiandin mit Gesten verzückten Schreckens erblickt wird. Darwins Gedicht stützt sich übrigens auf einen Text, der auch die Vorlage für Reinhold und Schiller bildete: die Darstellung der antiken Mysterien in William Warburtons neunbändigem Werk The Divine Legation of Moses ${ }^{46}$.

Wir lernen aus der Ikonographie des verschleierten Bildes zu Sais, worum es in der Gleichsetzung von Isis und Jahweh geht. Die ägyptische Göttin Isis gilt allgemein als Personifikation der Natur, und zwar in ihrem unenträtselbaren, aus sich selbst und von innen bewegten Aspekt der natura naturans, als beseelter Organismus und nicht als von außen bewegte Maschine ${ }^{47}$. Die Gleichsetzung von Jahweh mit Isis bedeutet daher nichts anderes als eine Paraphrase von Spinozas Deus sive Natura ${ }^{48}$.

Im 17. und 18. Jahrhundert wurde die Lehre Spinozas mit Atheismus und Materialismus assoziiert. Angemessener wäre der Begriff 'Kosmotheismus', denn in der Tat greift Spinoza die antike Vorstellung des von innen beseelten und bewegten Kosmos auf. ${ }^{49}$ Damit steht er einerseits in der Tradition von Giordano Bruno ${ }^{50}$ und Giulio Cesare (Lucilio) Vanini ${ }^{51}$, andererseits aber

45 Irwin Primer: Erasmus Darwin's Temple of Nature: Progress, Evolution, and the Eleusinian Mysteries. In: Journal of the History of Ideas, Vol. 25 (1964), No. 1, S. 58-76.

46 William Warburton: The Divine Legation of Moses Demonstrated on the Principles of a Religious Deist, from the Omission of the Doctrine of a Future State of Reward and Punishment in the Jewish Dispensation. London 1738-1741 (2. Aufl.: 1778).

47 Vgl. hierzu Robin G. Collingwood: The Idea of Nature. Oxford: Clarendon Press 1945.

48 Reinhold trug sich in den Jahren 1786-87, als er die Hebräischen Mysterien schrieb, mit dem Plan einer Dissertation über Spinoza, vgl. Anm. 56.

49 Vgl. Anm. 28.

50 Vgl. auch Giordano Bruno: The Expulsion of the Triumphant Beast. New Brunswick/NJ: Rutgers University Press 1964, S. 240: "So, then, that God, as absolute, has nothing to do with us except insofar as he communicates with the effects of Nature and is more intimate with them than Nature herself. Therefore, if he is not Nature herself, he is certainly the nature of Nature, and is the soul of the Soul of the world, if he is not the Soul herself." Vgl. auch Jan Bialostocki: The Renaissance Concept of Nature and Antiquity. In: The Message of Images. Studies in the history of Art. Vienna: IRSA Verlag 1988, S. 64-68, bes. S. 68 mit Anm. 51-54.

51 De admirandis Naturae reginae deaeque mortalium arcanis, Dialogus L, De Deo. In: Le opere di Giulio Cesare Vanini e le loro fonti di Luigi Corvaglia. Milano u. a.: Dante Alighiere 1934, Bd 2, S. 276: "In unica Naturae Lege, quam ipsa Natura, quae Deus est [est enim principium motus] in omnium gentium animis inscripsit" (zit. nach S. Berti: II Tratta- 
auch in jüdischen, insbesondere - aber nicht ausschließlich ${ }^{52}$ - kabbalistischen Traditionen. Die Frage einer möglichen Abhängigkeit Spinozas von R. Abraham Cohen Herreras kabbalistischer Schrift Pforte des Himmels wurde schon im 18. Jahrhundert lebhaft erörtert ${ }^{53}$. Reinhold erwähnt Spinoza zwar nicht. Er schreibt sein Buch aber auf dem Höhepunkt der Spinozarenaissance ${ }^{54}$, die 1785 durch Friedrich Heinrich Jacobis "Spinoza-Büchlein" ausgelöst wurde ${ }^{55}$, und trug sich während dieser Zeit mit dem Plan einer Dissertation über Spino$\mathrm{za}^{56}$. Man geht daher sicher nicht fehl, wenn man seine Schrift als eine Ehrenrettung Spinozas versteht, die in der Gestalt der Isis Spinozas Gottes- bzw. Naturbegriff über alle möglichen kabbalistischen und neoplatonischen Zwischenglieder hinweg unmittelbar mit der biblischen Selbstvorstellung Jahwehs in Einklang bringen will.

\section{Einheit und Namenlosigkeit - Hen kai Pan}

Das Schauervolle und Erhabene der Einweihung liegt für Schiller und Reinhold darin, daß die Grenzen der Sprache überschritten werden. Alle sprachli-

to dei tre impostori, S. 272. Vgl. Margaret C. Jacob: The Radical Enlightenment. Pantheists, Freemasons and Republicans. London: Allen \& Unwin 1981 (Early Modern Europe Today), S. 39. In dem älteren Traktat De tribus Impostoribus (der mit den Worten beginnt "Deum esse") lesen wir "hoc Ens [...] alii naturam vocant, alii Deum" (Wolfgang Gericke: Das Buch "De Tribus Impostoribus". Berlin: Evangelische Verlags-Anstalt 1982 [Quellen; Neue Folge, H. 2], S. 61, § 7)

52 Vgl. Moshe Idel: Deus sive natura - Les métamorphoses d'une formule e Maimonide à Spinoza. In: Maimonide et la mystique juive. Übers. Ch. Morsik. Paris: Éditions du Cerf 1991, S. 105-136.

53 Scholem, Die Wachtersche Kontroverse (wie Anm. 28); ders., Abraham Cohen Herrera Leben, Werk und Wirkung. In: Das Buch Scha ${ }^{\mathrm{C}}$ ar ha-Schamajim oder Pforte des Himmels in welchem die kabbalistischen Lehren philosophisch dargestellt und mit der Platonischen Philosophie verglichen werden, von Rabbi Abraham Cohen Herrera, dem Portugiesen. Aus dem Lateinischen übersetzt von Friedrich Häußermann. Frankfurt a. M.: Suhrkamp 1974 (Theorie), S. 7-67. - Ich verdanke den Hinweis auf diese Publikation Moshe Barasch. Horst Folkers: Das immanente Ensoph. Der kabbalistische Kern des Spinozismus bei Jacobi, Herder und Schelling. In: Kabbala und Romantik. Hg. von Eveline Goodman-Thau, Gerd Mattenklott und Christoph Schulte. Tübingen: Niemeyer 1994 (Conditio Judaica. Studien und Quellen zur deutsch-jüdischen Literatur- und Kulturgeschichte, 7), S. 71-95.

54 Vgl. Timm, Gott und die Freiheit (wie Anm. 28), Bd I: Die Spinozarenaissance.

55 Jacobis Spinoza Büchlein nebst Replik und Duplik. Hg. von Fritz Mauthner. München: Müller 1912 (Bibliothek der Philosophen, 2); Die Hauptschriften zum Pantheismusstreit zwischen Jacobi und Mendelssohn. Hg. und mit einer historisch-kritischen Einleitung versehen von Heinrich Scholz. Berlin: Reuther \& Reichard 1916 (Neudrucke seltener philosophischer Werke, 6); Alexander Altmann: Lessing und Jacobi. Das Gespräch über den Spinozismus. In: Lessing Yearbook, Bd 3 (1971), S. 25-70. Vgl. auch Karl Christ: Jacobi und Mendelssohn. Eine Analyse des Spinozastreits. Würzburg: Königshausen und Neumann 1988.

56 Fuchs, Karl Leonhard Reinhold (wie Anm. 29), S. 155, Anm. 115. 
chen Beschreibungen, Erklärungen und Benennungen fallen weg. Auf der letzten Stufe der Einweihung hört die Belehrung auf und überläßt den Initianden der reinen, unmittelbaren Schau (Epoptie). Die hierfür immer wieder herangezogene Quelle ist Clemens von Alexandrien:

Die Lehren, die in den Großen Mysterien erteilt werden, betreffen das Universum. Hier endet alle Unterweisung. Die Dinge werden geschaut wie sie sind, und Natur, sowie die Werke der Natur, werden erblickt und begriffen. ${ }^{57}$

Die Schau übersteigt alle Möglichkeiten diskursiver Erörterung. Zur Sprachlosigkeit der letzten Erkenntnis gehört auch der Entzug aller Namen für den Einen. Reinhold hatte gezeigt, daß das hebräische Tetragramm und seine Auslegung in Ex 3,14 nicht die Offenbarung, sondern die Verweigerung eines Namens bedeuten, oder vielmehr die Offenbarung des namenlosen Gottes. In dieser Namenlosigkeit sieht Schiller das Erhabene des Gottesbegriffs, den die ägyptischen Mysterien bewahren und vermitteln:

Die Epopten erkannten eine einzige höchste Ursache aller Dinge, eine Urkraft der Natur, das Wesen aller Wesen. Nichts ist erhabener, als die einfache Größe, mit der sie von dem Weltschöpfer sprachen. Um ihn auf eine recht entscheidende Art auszuzeichnen, gaben sie ihm gar keinen Namen. Ein Name, sagten sie, ist bloß ein Bedürfnis der Unterscheidung, wer allein ist, hat keinen Namen nötig, denn es ist keiner da mit dem er verwechselt werden könnte. ${ }^{58}$

Schiller paraphrasiert einen Satz des Hermes Trismegistus, der bei Laktanz überliefert ist. Schon Warburton und Reinhold hatten diese Stelle herangezogen. Es lohnt sich, sie im Zusammenhang zu betrachten.

Dieser [Trismegistus] schrieb Bücher - und zwar sehr viele, die sich auf das Wissen von den göttlichen Dingen beziehen - in denen er für die Erhabenheit des Höchsten und Einzigen Gottes eintritt, und er nennt ihn mit denselben Namen, die auch wir gebrauchen: Herr und Vater. Damit niemand auf den Gedanken verfällt, nach seinem Namen zu fragen, sagt er: Er ist 'ohne Namen'; er bedarf der Kennzeichnung durch einen Eigennamen nicht aufgrund seiner Einzigkeit. Dies sind seine Worte: 'der Eine Gott', 'der Gott der keines Namens bedarf', 'Er ist der namenlose Seiende'. Daher hat Gott keinen Namen, weil er der Einzige ist, und es keiner besonderen Kennzeichnung bedarf wo nicht eine Vielheit Unterscheidung erfordert, so daß du jeden einzelnen durch seine ihm eigene Kennzeichnung und Benennung identifizieren kannst. Für Gott aber ist der rechte Name 'Gott' weil er immer nur Einer ist. ${ }^{59}$

57 Clem. Alex. Strom. V.XI.17,1. Warburton I, 191.

58 Schiller, Die Sendung Moses (wie Anm. 38), S. 745.

59 Lactantius: Div. Inst. I 6; Laktanz paraphrasiert den hermetischen Traktat Asclepius 20, hg. Nock und Fetugrère, Corpus Hermeticum II, S. 320f.; Vgl. Reinhold, Die Hebräischen Mysterien (wie Anm.31), S. 54: Hic [Trismegistus] scripsit libros, in quibus majestatem summi ac singularis Dei asserit, iisdemque nominibus appellat, quibus nos Deum et patrem, ac ne quis NOMEN ejus requireret, ANONYMON esse dixit, eo quod Nominis proprietate non egeat, ob ipsam scilicet UNITATEM. Ipsius verba sunt: Deo igitur Nomen 
Schon um die Mitte des 15. Jahrhunderts - diesen Fund verdanke ich Aleida Assmann -, einige Jahrzehnte also bevor Hermes Trismegistus durch Marsilio Ficinos lateinische Ausgabe des Corpus Hermeticum (1471) zu einer Zentralfigur der Renaissance wurde, griff Nikolaus von Cues diese Stelle auf und schrieb:

Es ist ja einleuchtend, daß kein Name eigentlich dem Größten angemessen sein kann, da es das schlechthin Größte ist, zu dem nichts in Gegensatz tritt. Alle Namen sind nämlich aufgrund einer gewissen Besonderheit in der verstandesmäßigen Erfassung den Dingen zugelegt, auf der die Unterscheidung des einen vom anderen beruht. Wo jedoch alles eines ist, da kann es keinen besonderen Namen geben. Hermes Trismegistus sagt darum mit Recht: Da Gott die Gesamtheit der Dinge ist, so gibt es keinen ihm eigenen Namen, müßte doch sonst Gott mit jeglichem Namen benannt werden oder alles mit seinem Namen. Er umgreift ja in seiner Einfachheit die Gesamtheit aller Dinge. Gemäß seinem eigentlichen Namen der für uns als unaussprechbar gilt und das Tetragramm ist, d. h. aus vier Buchstaben besteht, und der deshalb eigentümlicher Name ist, weil er Gott nicht zukommt gemäß irgendeiner Beziehung zu den Geschöpfen, sondern gemäß seinem eigenen Wesen - müßte man ihn deuten als 'Einer und Alles' oder 'Alles in Eins', was noch besser ist ['unus et omnia' sive 'omnia uniter', quod melius est]. So haben wir oben auch die größte Einheit gefunden, die dasselbe ist wie 'Alles in Eins'. Freilich noch gemäßer und zutreffender als 'Alles in Eins' erscheint der Name 'die Einheit' [unitas]. Deshalb sagt der Prophet, daß "an jenem Tage Gott Einer sein wird und sein Name das Eine" und an anderer Stelle "Höre Israel, dein Gott ist 'Einer'."60

Diese Gleichsetzung des hermetischen unus et omnia bzw. Hen kai pan mit dem ahad bei Zacharja 14,9 und Deuteronomium 6,5 ist ebenso verblüffend und kühn wie Reinholds Gleichsetzung von 'Der ich bin' (ascher ahjaeh) und 'was da ist' (to esomenon). Sie versteht das ahad nicht als Zahlangabe - 'nur Einer ist Gott, und Gott hat nur Einen Namen' - sondern als Name: Gott heißt 'Eins' oder unitas. Wir erinnern uns, daß auch Isis in den Isidorus-Hymnen 'die Eine' genannt wird (ägyptisch in griechischer Transkription: THIOUIS $=\mathrm{t}_{3} \mathrm{w}^{\mathrm{c}} \mathrm{t}$ ). Warburton zitiert zwar Cusanus nicht, aber er wird diese Stelle gekannt haben, denn die Verbindung des von Laktanz überlieferten hermetischen Texts mit Zacharja 14,9 begegnet auch bei ihm.

Laktanz hielt Hermes Trismegistus für einen ägyptischen Weisen zumindest gleichen Alters wie Moses und für eine theologische und philosophische Autorität allerersten Ranges, die er auf Schritt und Tritt zitiert. Die Wiederentdeckung der hermetischen Schriften im Westen führte bekanntlich zu einer Renaissance des Trismegistus, die geradezu umwälzende Folgen für die abend-

non est, quia solus est: nec opus est proprio vocabulo nisi cum discrimen exigit MULTITUDO, ut unamquamque personam sua nota et appellatione designes.

60 Nikolaus von Kues: De docta ignorantia / Die belehrte Unwissenheit. Übersetzt und mit Vorwort und Anmerkungen hg. von Paul Wilpert. Hamburg: Meiner 1993 (Schriften des Nikolaus von Kues, 15. Philosophische Bibliothek, 264), Bd 1, Kap. XXIV, S. $96 f$. 
ländische Philosophie hatte. Gewöhnlich hält man diese Blütezeit des Neuhermetismus mit dem Jahre 1614 für beendet, als Isaak Casaubon die späte Entstehungszeit der hermetischen Schriften nachwies und das ganze Corpus Hermeticum für eine christliche Fälschung erklärte. Dabei wird jedoch übersehen, daß Ralph Cudworth, einer der Cambridge Platonists und Professor Regius für Hebräisch, in voller Anerkennung der Casaubonschen Kritik das Corpus Hermeticum als Quelle altägyptischer Theologie voll und ganz rehabilitiert hatte. Sein Buch The True Intellectual System of the Universe erschien in London $1678^{61}$ und wurde bis ins 19. Jahrhundert hinein immer wieder aufgelegt. Johann Lorenz von Mosheim besorgte in Deutschland eine erweiterte Ausgabe in lateinischer Übersetzung. Cudworths Bild der hermetischen Philosophie war im Denken des 18.Jahrhunderts zweifellos wesentlich präsenter als Casaubons Entlarvung. In diesem Buch hatte Cudworth sämtliche Stellen des Corpus Hermeticum sowie eine Fülle weiterer Belege zusammengetragen, die sich auf den Begriff des All-Einen beziehen und die ihn in Wendungen wie Hen to Pan, Hen kai Pan usw. umschreiben. Cudworth erblickt in der Lehre der Alleinheit den Inhalt der ägyptischen 'Arcantheologie'. Orpheus, der sich in die ägyptischen Mysterien habe einweihen lassen, habe sie nach Griechenland gebracht, wo Heraklit, Parmenides und Plato sie zur Philosophie ausgestaltet hätten. Moses, ebenfalls eingeweiht in die ägyptischen Mysterien, habe die ägyptische Arcantheologie den Hebräern vermittelt.

61 Ralph Cudworth: The True Intellectual System of the Universe: the First Part, wherein All the Reason and Philosophy of Atheism is Confuted and its Impossibility Demonstrated. London 1678 [2. Aufl.: 1743]. 